\title{
Functionally defective high density lipoprotein is pro-oxidant : a deviation from normal atheroprotective character
}

\author{
S. Sini. , N. Jayakumari \\ Dept.of Biochemistry, Sree Chitra Tirunal Institute for Medical Sciences and Technology, Thiruvananthapuram, Kerala,India
}

Email address:

jaya@sctimst.ac.in (N. Jayakumari)

To cite this article:

S. Sini. , N. Jayakumari. Functionally Defective High Density Lipoprotein is Pro-Oxidant: a Deviation from Normal Atheroprotective Character, International Journal of Nutrition and Food Sciences. Vol. 2, No. 3, 2013, pp. 92-101. doi: 10.11648/j.ijnfs.20130203.11

\begin{abstract}
High-density lipoprotein is a potential life saving antiatherogenic molecule. However, not all HDL is functionally similar, it can become dysfunctional and may increase atherosclerotic risk. At present, it is unknown, which structural alterations of HDL are essential accounting for its defective functionality and the precise pro-atherogenic mechanisms of action. This study is aimed at identification of the possible prevalence of dysfunctional HDL in subjects and its compositional and functional characterization in comparison to that of functional HDL. HDL was isolated from serum by ultracentrifugation and subjected to functional assays. HDL from majority of healthy subjects showed remarkable antioxidant property by inhibiting LDL oxidation. However, in those healthy subjects with systemic oxidative stress and inflammatory response as well as in those with known coronary heart disease, HDL was dysfunctional and promoted LDL oxidation. Dysfunctional HDL was truly pro-oxidant as it induced intracellular reactive oxygen species formation in cultured monocytes/macrophages. Functional deficiency in HDL did not show any association with HDL-cholesterol content. However, its characterization showed an enrichment of triglycerides, phospholipids, lipid peroxides, and diminished activity of paraoxonase-1, compared to functional HDL, which might render the particle dysfunctional and pro-oxidant.This study demonstrates the prevalence of dysfunctional HDL even among healthy subjects, despite normal HDL-C level, and in majority of subjects with known CHD, which is pro-oxidant in nature that might promote vascular inflammation and atherogenesis. The functional assay of HDL could lead to improved predictive accuracy of cardiovascular disease risk associated with circulating HDL.
\end{abstract}

Keywords: Atherosclerosis, Inflammation, HDL-Function, Pro-Oxidant

\section{Introduction}

Cardiovascular disease has emerged as a major health burden in many developing countries, including India. It has been reported that coronary heart disease [CHD] is more common in urban than rural areas of India[1] and it affects people at younger ages. Recent research has shown that atherosclerosis, the main cause of CHD, is an inflammatory disease in which immune mechanisms interact with metabolic risk factors to initiate lesions in the arterial tree[2]. CHD has a multi-factorial etiology, with many of the risk factors being influenced by lifestyle. Several prospective epidemiological studies have found that low level of serum high-density lipoprotein-cholesterol (HDL-C) constitute a strong, independent risk factor for coronary heart disease[3-5]. HDL protects against atherosclerosis by regulating cholesterol efflux from tissues, modulating in- flammation, providing antioxidant, vasoprotective, antithrombotic and anti-infectious properties[6]. However, many patients who experience a clinical event have normal or even high levels of HDL-C[7]. Recent studies indicate that higher HDL levels may not always be protective and HDL can become functionally deficient under conditions of acute phase response, atherogenic dyslipidemia and insulin resistance[6, 8, 9]. These findings indicate that an altered form of atheroprotective HDL, i.e. proinflammatory HDL, may also increase atherosclerotic risk. HDL particles are quite heterogeneous, encompassing a range of sizes, densities and contain multiple surface apolipoproteins, varying concentrations of cholesterol and phospholipids, and various antioxidant or pro-oxidant enzymes, which are now recognized as significant factors in the balance of pro- and antiatherogenic metabolism[8].

Even though there are several evidences showing the 
prevalence of dysfunctional HDL[9-11] in patients with acute coronary syndromes, the underlying pathways through which HDL might get converted into a proinflammatory stage are quite unknown. Available data identified enrichment of HDL with myeloperoxidase, triglycerides, group IIA secretory phospholipase A2, ceruloplasmin, serum amyloid $\mathrm{A}$ and haptoglobin-hemoglobin complex, can attenuate the antiatherogenic capacity of HDL[6, 12]. Since HDL represents a heterogeneous molecule any alteration occurring in its composition and or structure may impair its biological function and convert it into a pro-inflammatory molecule. A recent study from this laboratory has demonstrated that in vitro oxidative modification of HDL loses its atheroprotective functions and exerts proinflammatory response and oxidative stress in human monocytes/macrophages [13].The generation of oxidized HDL and/or dysfunctional HDL(dysfunHDL) in vivo might therefore be regarded as possibly atherogenic. There is not enough clinical data to support this concept of functional deficiency in HDL as a contributing factor for the increased prevalence of CHD.

Earlier study from this laboratory revealed that serum levels of HDL-C and paraoxonase-1, an antioxidant enzyme associated with HDL, showed inverse association with the presence of CHD but were not related to the severity of disease in terms of number of diseased vessels[14].It is not clear that measuring plasma concentrations of HDL$\mathrm{C}$ are sufficient to capture the functional variations in HDL particles along with the associated cardiovascular risk. Therefore, it is highly essential to evaluate functional characteristics of HDL, such as antioxidant and antiinflammatory functions as well as HDL-associated biomarkers that better predict cardiovascular risk. An understanding of the molecular mechanisms of HDL action may provide important insights into the development of new therapeutic advances targeted towards HDL for further reducing the atherogenic cardiovascular risk.

Many studies have suggested that traditional cardiovascular risk factors alone cannot explain the excess risk for cardiovascular diseases $[15,16]$ and non-traditional cardiovascular risk factors may also play a major role.There is increasing recognition that chronic subclinical vascular inflammation plays a role in the pathogenesis of atherosclerosis[17, 18]. Rapid CHD progression in patients with stable angina pectoris has been shown to be associated with increased C-reactive protein [CRP] levels and raised concentrations of biochemical markers of endothelial and macrophage activation[19]. Cellular adhesion molecules (CAMs) and matrix metalloproteinases (MMPs) have also been suggested to represent an important factor in atheromatous plaque disruption and the occurrence of acute coronary syndromes[20]. These observations suggest that monocyte/macrophage activation plays a pathogenic role in plaque vulnerability and accelerated stenosis progression. HDL appears to inhibit inflammation associated with atherosclerotic plaque development and regress atherosclerosis. HDL-replacement therapy is a promising new treatment strategy involving the acute administration of HDL to rapidly stabilize patients at imminent risk for developing a myocardial infarction, such as those with acute coronary syndrome[21].

With this background we hypothesized that harmful functional alteration in HDL, could contribute to enhanced atherogenesis and trigger atherosclerotic-lesion progression directly or indirectly through various cellular messengers. In view of this we aimed to analyze the functional properties of HDL isolated from apparently healthy volunteers to adequately assess whether the circulating HDL is cardioprotective and compared the results with those having known CHD. We investigated the functional alteration in HDL in terms of its ability to inhibit LDL oxidation, and the association of HDL function with lipid profile, systemic markers of inflammation, oxidative stress and HDLassociated biomarkers. Further, we studied the influence of functional and dysfunctional HDL on monocyte/macrophage function relevant to atherogenesis (eg. Cellular oxidative stress) using cell culture model.

\section{Methods}

\subsection{Materials}

Histopaque 1077, RPMI 1640, Penicillin, Streptomycin, Gentamicin, Dichloro- fluoresceine diacetate (DCFH-DA), Trypan blue and Potassium bromide, were purchased from Sigma Chem. (St. Louis, USA). Cell culture dishes were procured from NUNC (Denmark).

\subsubsection{Sample collection}

Fasting blood samples were collected from apparently healthy volunteers (both both males and females) of this hospital staff [ 40] with institutional review board approval and informed consent. Serum was collected by low speed centrifugation and subjected to various biochemical analyses. Samples collected from few patients with known coronary heart disease [ 20] were also included in this study for comparison of HDL function.

\subsection{Analytical Methods}

\subsubsection{Serum Lipids}

Serum total cholesterol and triglycerides[TG]were estimated by the recommended enzymatic assay methods CHOD-POD and GPO-POD respectively using enzyme assay kits [Helix India]. HDL-cholesterol was quantitated by polyethylene glycol [PEG] method as described by Izzo et al[22]. LDL-cholesterol was calculated using the Friedewald's formula as LDL-C=[total cholesterol-(HDL$\mathrm{C}+\mathrm{TG} / 5) \mathrm{mg} \%$. Serum phospholipid was assessed using recommended reagent kits (Pamed service,New Delhi).All the assays were carried out in semi autoanalyser (Nexgen auto analyzer].

\subsubsection{Oxidative Stress Markers in Serum}

Lipid peroxides and protein carbonyls were quantitated by thiobarbituric acid method using malondialdehyde as 
standard[23] and protein carbonyls using dinitrophenyl hydrazine(DNPH) respectively[24]. Antioxidant vitamin C was quantitated by recommended spectrophotometric method[25], which depended on the reduction of ferric to ferrous ion by ascorbic acid and the ferrous ion was determined as the red-orange $\alpha, \alpha$-dipyridyl chromophore at 525 $\mathrm{nm}$ using UV-VIS-1601 spectrophotometer (Shimadzu).

\subsubsection{Inflammatory Markers in Serum}

hs-CRP was measured by ELISA method as described by manufacturer's instruction( Cayman Chemicals,USA) and gelatinase activity based on gelatin zymography[26].

\subsubsection{Isolation of Lipoproteins}

HDL $[\mathrm{d}=1.063$ to $1.21 \mathrm{~g} / \mathrm{ml}]$ and LDL[ $\mathrm{d}=1.006$ to 1.063 $\mathrm{g} / \mathrm{ml}$ ] were isolated from serum by standard sequential density gradient ultracentrifugation[27] in a Beckman Optima TLX 120 Ultracentrifuge with a fixed angle rotor. The isolated lipoproteins were desalted on PD-10 columns equilibrated with PBS buffer, $\mathrm{pH} 7.4$ containing $0.15 \mathrm{M} \mathrm{NaCl}$. The purity of the isolated lipoproteins was confirmed by polyacrylamide gel [3.75\%] electrophoresis and the protein content was estimated by Lowry's method[28].

\subsubsection{Characterization of Isolated $\mathrm{HDL}$}

HDL content of cholesterol, triglycerides, phospholipids, and lipid peroxides were quantitated as described above. Paraoxonase-1 (PON-1) activity was measured using phenyl acetate as substrate[29]

\subsubsection{Assay of Antioxidant Capacity of HDL}

HDL was tested for its ability to inhibit the oxidation of control LDL [pooled LDL, isolated from healthy subjects having normal lipid profile]. The functionality of HDL was assessed in terms of the antioxidant ability of HDL to prevent the formation of the fluorescence signal generated by oxidized LDL[30]. Briefly an aliquot of LDL was subjected to air-oxidation for 2 hours in the presence and absence of HDL and then treated with DCFH-DA(10 $\mu$ l of $2 \mathrm{mg} / \mathrm{ml}$ stock) for 1.5 hours. The resultant fluorescence was measured at an excitation wavelength of $485 \mathrm{~nm}$ and an emission wavelength of $530 \mathrm{~nm}$ using Fluorescence Eliza Plate Reader (Biotek FLX 800).

\subsubsection{Monocyte Cell Isolation and Culture}

Human peripheral blood mononuclear cells were isolated from blood of healthy volunteers by Histopaque 1077 (sigma Aldrich,USA) based density gradient centrifugation. The buffy coat formed at the interface was collected, washed twice with PBS pH 7.4, and finally with RPMI 1640 medium (sigma Aldrich,USA). The pellet was resuspended in RPMI medium. Cells $\left(1 \times 10^{6} / \mathrm{ml}\right)$ were then seeded on to culture dishes and incubated for $2 \mathrm{~h}$ for adherence in an atmosphere of $5 \% \mathrm{CO}_{2}$ at $37^{\circ} \mathrm{C}$. Non-adherent cells were removed by washing and the monocytes adhered to dishes were maintained in serum free RPMI 1640 medium supplemented with penicillin (100 U/I), streptomycin (100 mg/l), and gentamicin (100 mg/l) for $24 \mathrm{~h}$. Cell viability was assessed by Trypan blue exclusion test and was found to be greater than $95 \%$. These cells represent monocytes in an early stage of macrophage differentiation and are thus referred to as monocytes-macrophages in the text[31].

\subsubsection{Cell Treatment}

Monocytes/macrophages were maintained in culture as described above and then treated with a medium containing PBS alone, with functional HDL[funHDL] or dysfunctional HDL[dysfunHDL] at different concentrations [100 to $700 \mu \mathrm{g} / \mathrm{ml}$ ] for 24 hours.Cell culture supernatants were collected and cells were dislodged by $3 \mathrm{mM}$ EDTA treatment and total cell protein was determined by Lowry's method[28].

\subsubsection{Measurement of Intracellular ROS}

Intracellular reactive oxygen species[ROS] were measured by DCFH method[30]. Monocytes/macrophages after treatment with HDL were incubated with DCFH-DA in medium at $37^{\circ} \mathrm{C}$ for $45 \mathrm{~min}$. After washing with $\mathrm{PBS}$, DCFH fluorescence of the cells from each well was measured in a fluorescence microplate reader (Biotek FLX 800) at an excitation wavelength of $485 \mathrm{~nm}$ and emission at 528 $\mathrm{nm}$. The intensity of fluorescence reflects the extent of oxidative stress.

\subsubsection{Statistics}

Statistical analysis was carried out using Graph Pad prism statistical software. One-way ANOVA was employed to analyze the variation among groups in the form of means. Differences between variables were also compared using Student's t test. The association between HDL-C and function was determined by calculating pearson's correlation coefficient ' $r$ '.'p'value of less than 0.05 was considered statistically significant.

\section{Results}

\subsection{Antioxidative Potential of $\mathrm{HDL}$}

To check whether antioxidative capacity of HDL was impaired in subjects, a fluorescent-based assay was performed, in which the HDL function was assessed in terms of the antioxidant capacity of HDL to prevent the formation of fluorescence signal generated by control LDL during oxidation. The results [Fig.1] showed that the antioxidative property of HDL (expressed as \% inhibition of LDL oxidation) varied widely among healthy subjects despite having similar HDL-C concentrations. For e.g. HDL isolated from four subjects with same concentration of low HDL-C of $32 \mathrm{mg} \%$ showed different antioxidant capacity as $30 \%, 47 \%, 55 \%$ and $60 \%$. A similar pattern was found for HDL functionality at different levels of HDL-C as indicated in Fig.1.It was also interesting to observe zero percentage $(0 \%)$ antioxidative capacity for HDL, among healthy subjects [in three cases] even after having normal serum HDL-C concentrations of 40,46 and $49 \mathrm{mg} / \mathrm{dl}$ indicating the prevalence of functionally deficient HDL. These 
findings indicated that the functional capacity of HDL varied among apparently healthy subjects irrespective of the levels of HDL-C and the levels of HDL-C did not show any relation to the antioxidative property of $\mathrm{HDL}(\mathrm{r}=0.13)$.

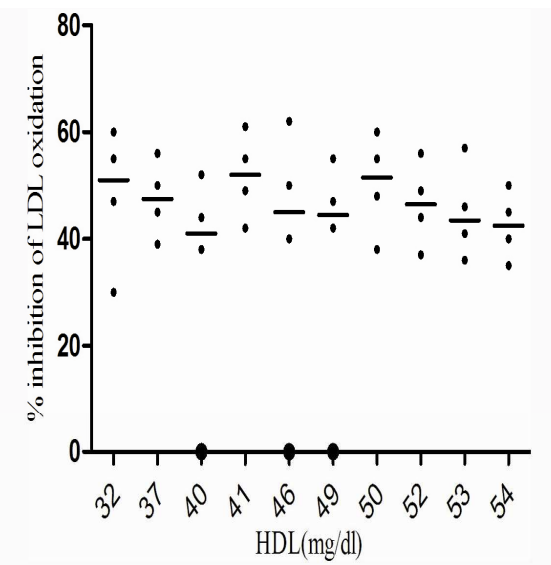

Fig 1: Antioxidative capacity of $H D L$.Antioxidative capacity of $H D L$ isolated from healthy volunteers.

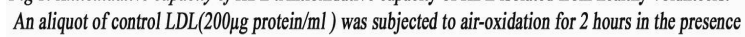

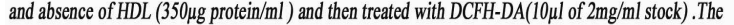
resultant fluorescence was measured at an excitation wavelength of $485 \mathrm{~nm}$ and an emission wavelength of $530 \mathrm{~nm}$ using Fluorescence Eliza Plate Reader. Antioxidative capacity of HDL are expressed as \% inhibition of LDL oxidation. Each dot in a row represents antioxidant capacity of four different $H D L$ fractions from subjects matched for HDL-Cand each horizontal line represents the mean antioxidant capacity of $H D L$

These healthy subjects were then divided into two groups according to the functionality of HDL, as having funHDL [group A], and dysfunHDL [group B]. To determine whether this form of dysfunctional HDL was also present in patients with known CHD, we assessed the functionality of HDL particles collected from these patients [group C] and compared with the healthy groups. It was observed that majority of healthy subjects [group A, mean HDL-C $=44.4 \pm 7.4]$ were found to have HDL with remarkable antioxidant capacity to inhibit LDL oxidation [mean antioxidant capacity of $\mathrm{HDL}=50 \% \pm 7.35]$. However, HDL isolated from a few healthy subjects [group B, mean HDL$\mathrm{C}$ of 3 subjects $=45 \pm 4.5 \mathrm{mg} / \mathrm{dl}]$ and majority of patients [group C, mean HDL-C $=36 \pm 4.9 \mathrm{mg} / \mathrm{dl}$ ) failed to inhibit LDL oxidation, i.e. antioxidant capacity of HDL was $0 \%$, suggesting the prevalence of dysfunctionality in HDL among apparently healthy subjects $[\sim 7.5 \%]$ and in most of the CHD patients. Further, the inflammatory index of HDL [iiHDL] was calculated for 20 pairs, where the subjects within each pair have same level of HDL-C but they were functionally different, i.e. funHDL and dysfun HDL. For this calculation, the fluorescence of oxidized LDL was used as an index [by normalizing LDL oxidation to 1.0] and examined the influence of HDL on this effect [32]. The proinflammatory HDL (piHDL) was defined by an HDL inflammatory index greater than 1.0 and that with less than 1.0 was defined as anti-inflammatory HDL [Fig.2]. FunHDL from group A healthy subjects showed an inflammatory index of $0.48 \pm 0.20$, indicating antiinflammatory nature. But, dysfunHDL as a group was found to be pro-inflammatory as evidenced by an inflammatory index of $1.88 \pm 0.48$.

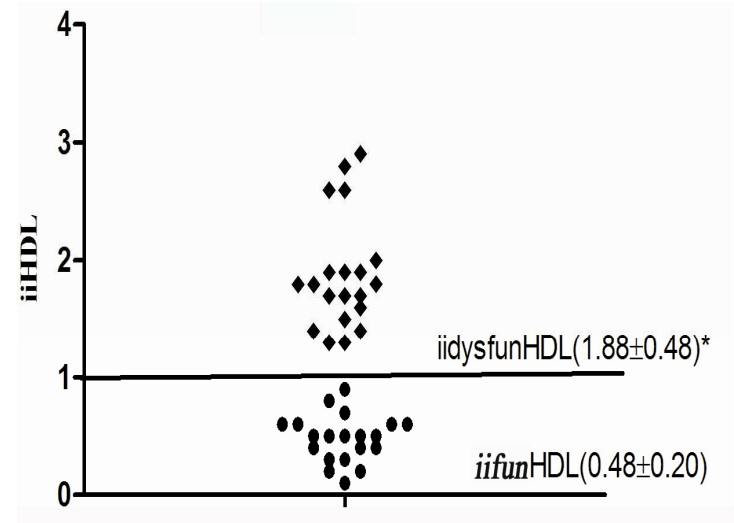

Fig 2: Inflammatory index of HDL(iiHDL). Inflammatory index of HDL(iiHDL) calculated using the fluorescence of oxidized $L D L$ as an index. [Normalizing $L D L$ oxidation to 1.0). Each dot represents iiHDL. The horizontal line at 1.0 indicates $L D L$ oxidation index. A value $>1.0$ is pro-inflammatory. ${ }^{*}$ FunHDL vs. dysfunHDL $p<0.05$

\subsection{HDL Functionality and Serum Lipid Parameters}

It is well established that an atherogenic lipid profile is associated with increased cardiovascular risk. To examine whether dysfunctionality in HDL showed any association with the lipid parameters, serum levels of total cholesterol, TG, LDL-C,HDL-C and phosohlipids were quantitated in these three groups of subjects and presented in Fig.3.Total cholesterol and LDL-C were significantly higher in group $B$ with dysfunHDL compared to group A[funHDL] and group $\mathrm{C}$ [dysfunHDL].In group $\mathrm{B}$ and $\mathrm{C}$ with dysfunHDL, a significant increase in the levels of TG and phospholipids was observed than that of group A with funHDL [ 'p'< 0.05].In the case of HDL-C, it was found lower[ ' $p$ ' $<0.05]$ only in group $\mathrm{C}$ patients with dysfunHDL. Among healthy subjects, no difference was observed in the mean levels of HDL-C between subjects with funHDL[group A] and dysfunHDL[group B].These results showed that subjects with dysfunHDL have higher levels of serum TG and phospholipids compared to those having funHDL.

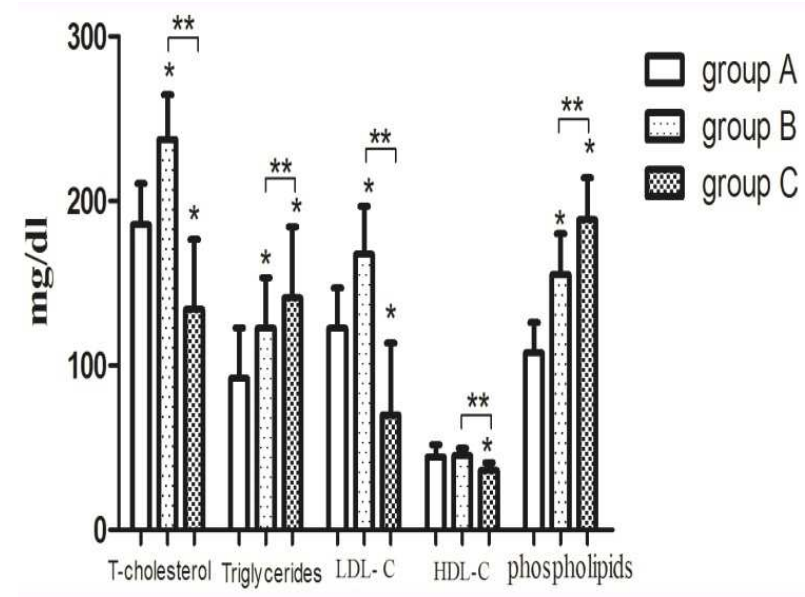

Fig.3. Lipid profile .Lipid profile of subjects with funHDL [group A healthy subjects] and dysfunHDL [group B healthy subjects, group C patients]. values are expressed as mean $\pm S D$. ${ }^{*}$ ' $p$ ' $<0.05 \mathrm{~A}$ vs. $B \& C$; ${ }^{* *}$ ' $p$ '< $0.05 B$ vs. $C$. 


\subsection{Dysfunctionality in HDL Associated with Systemic Oxidative Stress}

Oxidative stress represents an imbalance between prooxidants and anti-oxidants. HDL has an array of antiatherogenic mechanisms, which may prevent the formation and promote removal of lipid peroxides from other proatherogenic lipoprotein and cell membranes. It is not clear whether dysfunctionality in HDL has any relation with excess oxidative stress. To examine this, the serum concentration of lipid peroxides and protein carbonyls, the peroxidation products of lipids and proteins respectively, were quantitated in the three study groups of subjects. It was observed that the levels of both lipid peroxides and protein carbonyls were significantly higher in subjects with dysfunHDL [group B \& C] than that of funHDL, showing excess oxidative stress. ( Fig.4).In addition, serum content of antioxidant vitamin-C was found decreased in subjects with dysfunHDL and the difference was more significant in group C. [ vit. C content in group: $\mathrm{A}=1.38 \pm 0.41 ; \mathrm{B}=$ $0.83 \pm 0.27 ; \mathrm{C}=0.57 \pm 0.22 \mathrm{mg} / \mathrm{dl}, \mathrm{p}<0.01]$
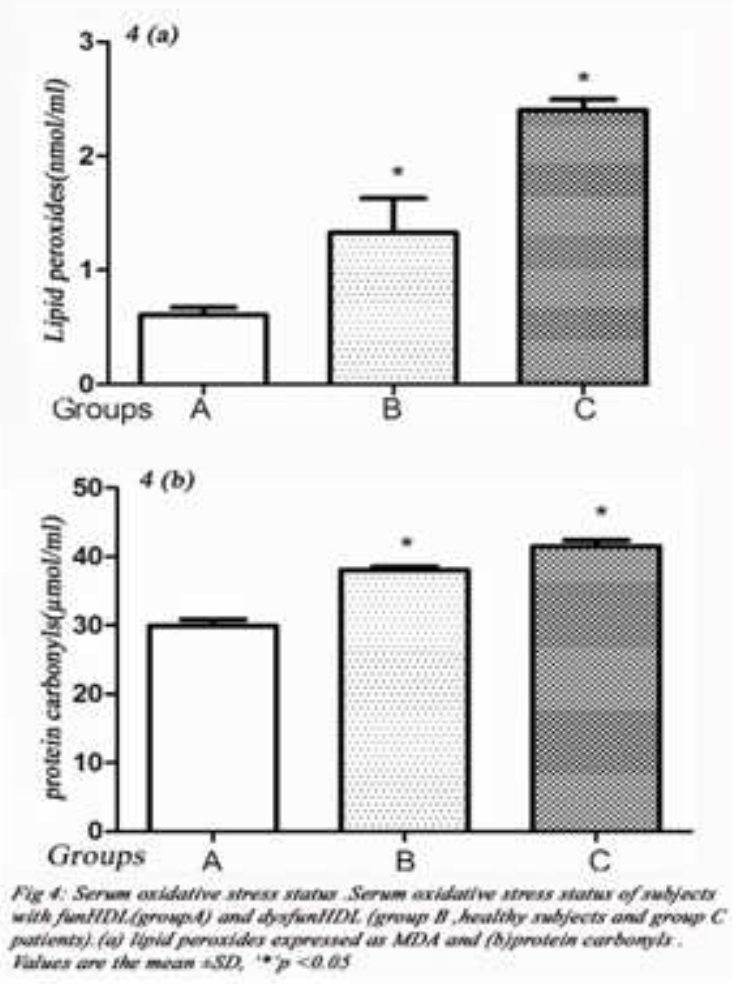

\subsection{Dysfunctionality in HDL and Inflammatory Status}

\subsubsection{Dysfunhdl \& Hs-CRP}

Inflammation has been recognized as a major contributor to atherogenesis through its effects on lipoprotein metabolism and arterial wall biology. To study the systemic inflammatory status, serum hs-CRP [ a strong inflammatory marker] was quantitated in these three groups of subjects and the results were presented in Fig.5.In subjects with dysfunHDL [group B \& C], we found significantly higher levels of hs-CRP compared to subjects with funHDL, indi- cating the presence of systemic inflammation in the former groups.

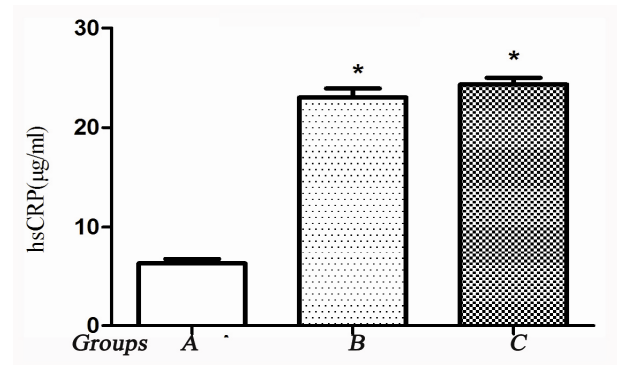

Fig 5: Serum inflammatory status. Circulating inflammatory marker. hsCRP levels in subjects with funHDL [group A] and dysfunHDL[group B: healthy subjects and group C: patients] .Values are expressed as mean $\pm S D .{ }^{*}$ ' $p$ '<0.05

\subsubsection{Dysfunhdl \& Mmps}

Matrix metalloproteinses [MMPs] play an important role in the homeostasis of extra cellular matrix and inflammation. They contribute both in the formation as well as in the destabilization of atherosclerotic plaque. Data about the relationship between alteration in HDL function and pattern of gelatinase expression in serum is limited. Therefore we assessed the two key members of MMP family-MMP-2 \& MMP-9[gelatinase -A \& -B] by gelatin zymography in these subjects. Gelatin zymography data of serum [fig.6] showed enhanced activity for both MMP-9 \& MMP-2 in subjects having dysfunHDL compared to those having funHDL.

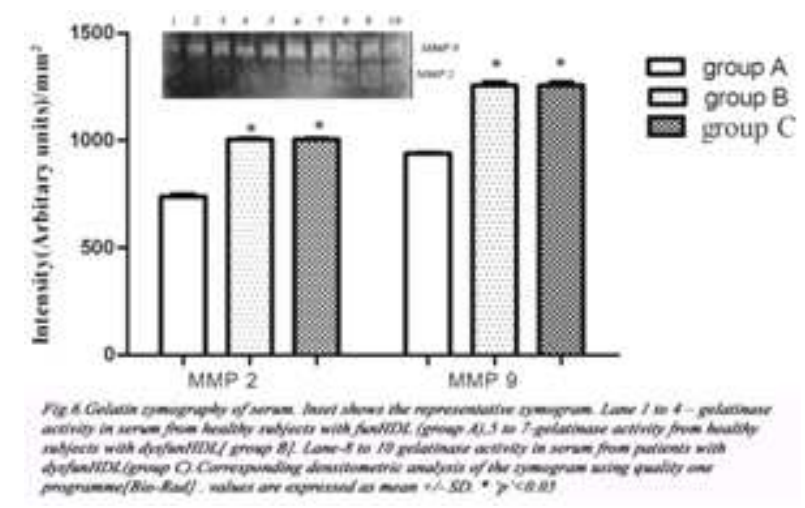

\subsection{Characterization HDL Particle}

HDL undergoes functional alteration in certain subjects. To determine the associated compositional changes, HDL fraction was isolated from serum by density gradient ultracentrifugation and subjected to chemical quantitation. The characteristics of isolated HDL particles were presented in Table I. The compositional analysis showed the enrichment of TG, phospholipids, and lipid peroxides in dysfunHDL[group B \& C] in comparison to funHDL. Cholesterol content of HDL particle was found decreased only in the dysfunHDL[group C] from patients. However, no difference was observed in the mean levels of cholesterol between funHDL and dysfunHDL [group A \& B] in healthy subjects. Further, dysfunHDL particles showed decreased activity of paraoxonase-1,an antioxidant enzyme and the 
difference was more significant in group $\mathrm{C}$, compared to funHDL [group A].The relative resistance of HDL particle to in vitro air-saline oxidation[2 hours] was also assessed by measuring the formation of ROS as DCFH fluorescence. A marked reduction in resistance to auto-oxidation was observed for dysfunHDL particle, as evidenced by enhanced ROS formation than the funHDL particle.

\begin{tabular}{|c|c|c|c|}
\hline \multicolumn{4}{|c|}{$\begin{array}{c}\text { TABLE I } \\
\text { Characterization of isolated HDL particle }\end{array}$} \\
\hline & $\begin{array}{l}\text { Group A } \\
\text { Subjects with } \\
\text { funHDL. }\end{array}$ & $\begin{array}{c}\text { Group } B \\
\text { Subjects with } \\
\text { dysfunHDL }\end{array}$ & $\begin{array}{r}\text { Group } C \\
\text { subjects with } \\
\text { dysfunHIDL }\end{array}$ \\
\hline \multicolumn{4}{|l|}{ PARAMETERS } \\
\hline Cholester ol [mg/d] & $40.6 \pm 7.3$ & $40.5 \pm 3.7 *$ & $30.8 \pm 6.3^{*}$ \\
\hline Triglyerides[mg/di] & $37.8 \pm 6.1$ & $67.8 \pm 4.1^{1 *}$ * & $86 \pm 7.8^{*}$ \\
\hline Phosholipids[mg/dl] & $55.3 \pm 8.8$ & $72.1 \pm 17.2^{*}$ & $82.6 \pm 7.5^{*}$ \\
\hline Paraoxonase-1[kul] & $137 \pm 23$ & $87 \pm 4.6^{* *}$ & $57 \pm 15.2^{*}$ \\
\hline Lipid peroxides(nm mit) & $0.06 \pm 0.02$ & $1.08 \pm 0.13^{*}$ & $1.08 \pm 0.09 *$ \\
\hline $\begin{array}{l}\text { Resistance to } \\
\text { in vitro oxidation } \\
\text { (ROS-fluorescence intensity) }\end{array}$ & $77 \pm 10.3$ & $112 \pm 8^{*} *$ & $136.2 \pm 12.16^{*}$ \\
\hline
\end{tabular}

\subsection{Dysfunhdl Induces ROS Production in Monocytes/ Macrophages}

To study the influence of dysfunHDL on monocyte function relevant to atherogenesis, human peripheral blood monocytes were isolated, cultured under standard conditions and treated separately with the funHDL and dysfunHDL for 24 hours. The intracellular formation of ROS was assessed based on DCFH fluorescence and presented in Fig. 7a \& b. Treatment of cells with dysfunHDL induced more ROS formation in a concentration- dependent manner, there by acting as a pro-oxidant whereas exposure of cells to funHDL prevented ROS formation and expressing its antioxidant property.

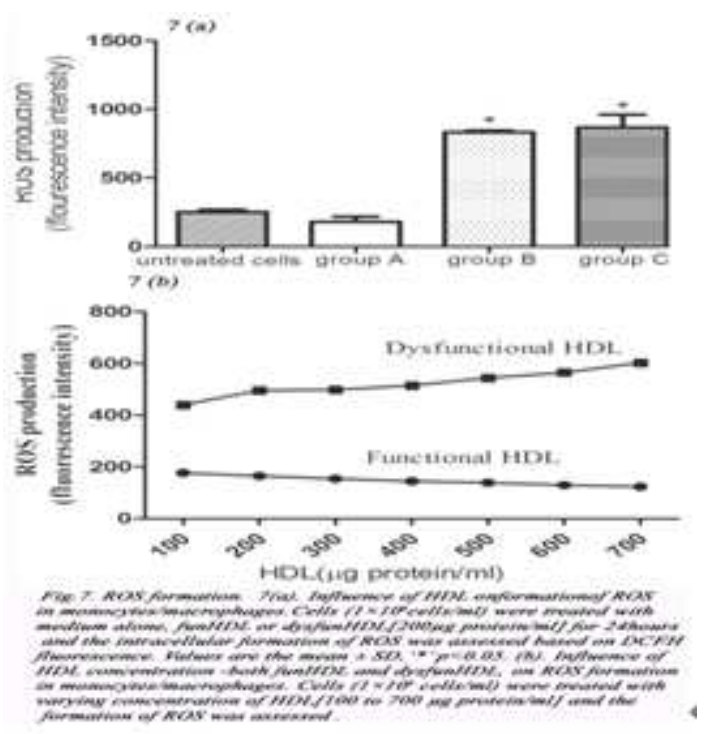

\section{Discussion}

Atherosclerosis can be considered as a chronic inflammatory disease driven by the progressive incorporation of the cytotoxic byproducts of lipid and phospholipid oxidation into a changing monocyte population at the cellular level of the arterial endothelium.[17]. High-density lipoproteins are recognized for their many atheroprotective functions[4]. It has also been implicated in the inhibition or the regression of atherosclerosis in humans and experimental animals[33].

The precise mechanisms involved in all these atheroprotective properties of HDL are only partially understood at the present time. The antioxidant activity of HDL protects and reverts oxidation of LDL, which is an early event in the formation of atherosclerotic lesions that promotes inflammation in the artery wall[34]. By limiting LDL oxidation, HDL plays a key anti-inflammatory role in slowing atherogenesis. In agreement to this, in the present study we observed that majority of healthy subjects express HDL with remarkable antioxidant capacity to inhibit LDL oxidation. However, HDL from a few healthy subjects $[\sim 7.5 \%$ of the study group], even if HDL-C is present in normal levels, failed to inhibit LDL oxidation indicating the prevalence of functionally deficient HDL among healthy subjects, similar to those observed in patients with CHD.The antioxidative capacity of HDL varied among apparently healthy volunteers irrespective of the levels of HDL-C. Since the rate of prevalence of dysfunHDL in our population is not known, more sample analyses for the functional assay of circulating HDL particle are needed to establish a firm database. However, this preliminary finding is in consistent with the reports of the prevalence of dysfunctional HDL in south Asian immigrants suffering from CAD $[9$, 10].The functionally deficient HDL was also found to be pro-inflammatory [inflammatory index of HDL > 1.0] by inducing LDL oxidation.It has also been reported that in human subjects with obstructive sleep apnea, HDL was significantly less able to retard LDL oxidation than HDL from control patients with similar HDL cholesterol levels[2]. Ansell et al. studied a cohort of patients with angiographic coronary disease and indicated that the inflammatory/anti-inflammatory properties of HDL distinguish patients with $\mathrm{CAD}$ or $\mathrm{CAD}$ equivalents from control subjects better than HDL cholesterol levels[35]. These findings support the general notion that functionally deficient HDL is not cardioprotective even if HDL-C is present in high levels. Characterization of HDL function i.e.proinflammatory HDL phenotype, could lead to improved predictive accuracy for CVD risk associated with circulating HDL.

HDL can undergo profound compositional and functional modifications in subjects with a variety of inflammatory and metabolic disorders with acute phase reactions and systemic inflammation, including systemic lupus erythematosus (SLE), CHD and other diseases causing systemic inflammation $[6,36]$.Acute phase response has both quan- 
titative and qualitative effects on HDL particle[37]. However, the underlying mechanism, which converts the normal HDL to a dysfunctional stage and its involvement in atherosclerosis development, are still unclear. We have shown that the antioxidative function of HDL was impaired in subjects with systemic inflammatory state as assessed by enhanced hs-CRP, and was found pro-inflammatory in nature as characterized by its enhanced capacity to induce LDL oxidation. hs-CRP is an acute-phase reactant protein whose plasma concentration increases in response to an inflammatory process anywhere in the body. Marked increase in the level of serum hsCRP, a srong marker of systemic inflammation, as well as enhanced activities of gelatinases [matrix metalloproteinases.,MMPs] were observed in subjects with dysfunHDL indicating a possible link between inflammation, oxidative stress and HDL dysfinctionality. Overexpression of MMPs has been implicated in the pathogenesis of various diseases, such as arthritis, atherosclerosis, and tumor invasion and metastasis[38]. MMPs contribute both in the formation as well as in the destabilization of atherosclerotic plaque and also predict the cardiovascular risk in patients with CAD[39].Furthermore, MMP-9[gelatinase-B] from macrophages and neutrophils is thought to play a key role in the migration of these cells during inflammatory diseases such as rheumatoid arthritis(RA) and also involved in the homeostasis of the extracellular matrix and known to play a key role in the development of hypertension[40]. Serum levels of MMPs are very likely influenced by release of MMPs following degranulation of leukocytes and platelets, and serve as a marker of pertinent intracellular protease activity. Alternatively elevated MMP concentration in serum might emanate from a circulating cell type associated with a pathological condition, suggesting that circulating leukocytes may be a source of MMP 9[41]. Recently an association between inflammation and arterial stiffness has been reported [42] and suggests a role for gelatinase in large artery stiffening which is associated with an increased CV risk. As such, better understanding of the dysfunctional aspects of HDL metabolism and gelatinase expression will become more important clinically and may also provide new strategies for its prevention/treatment.

HDL characterization indicated that dysfunctional HDL exhibited decreased activity of paraoxonase-1, HDLassociated antioxidant enzyme, and enrichment of lipid peroxides, triglycerides and phospholipids. Oxidation of phospholipids and apoproteins might occur within this dysfunctional HDL phenotype. HDL may acquire oxidized lipids from cells and by exchange with other particles, or HDL lipids may be oxidized in situ. The accumulated lipid peroxides and other oxidants in HDL can inhibit HDLassociated antioxidant enzymes, including paraoxonase-1, modify apoA1 and render the HDL particle unable to function as an effective antioxidant and convert it into a prooxidant form. In contrast, the antioxidant activity of functional HDL can prevent the formation of proinflammatory oxidized phospholipids, block the atherogenic activity of those proinflammatory molecules already formed[35]. This is mediated by the HDL-associated antioxidant enzymes and also via the direct action of apoA-I.HDL carries the bulk of lipid hydroperoxides in plasma.These lipid hydroperoxides can be converted into less reactive lipid hydroxides through specific methionine residues of apoAI and apoAII, with the concomitant formation of methionine sulfoxide. Fully funHDL may promote lipid hydroperoxide metabolism and its uptake and clearance by the liver, which would be protective[43]. In contrast, dysfunHDL might promote the transfer of lipid hydroperoxides to apoBcontaining lipoproteins and promote VLDL and LDL oxidation[8] that can increase vascular inflammation.

HDL particles are susceptible to structural modifications mediated by various mechanisms, including oxidation, glycation, or enzymatic degradation, affecting their functional properties[44]. Characterization of HDL particle in this study showed the enrichment of TG, phospholipids and lipid peroxides and diminished activity of PON-1,in dysfunHDL in comparison to funHDL. New findings $[45,46]$ are showing that apoA-I is also vital for HDL's enzyme functions that give it anti-inflammatory and antioxidant activity. An important indication that a person lacks apoA-I and has too much apoA-II is elevating triglycerides[47]. These findings are in agreement with Zago et al,who reported that that the degree of HDL functional loss correlated with its triglyceride (TG),but not cholesterol, content[48]. HDL lipid composition may be altered in atherogenic dyslipidemia and associated with inflammatotry state. An increase in HDL-TG content is frequently found in an acute phase reactant setting and as consequence of increase CETP activity[6].Elevated plasma TG levels represent another important factor that lowers HDL particles stability and plasma residence time, leading to functionally defective HDL[21].Thus, it is clear that HDL particles can vary in their atheroprotective capacity, and functionally deficient HDL may be involved with other atherogenic factors in the development of atherosclerosis. In support of this, we observed an association between dysfunctionalilty in HDL and higher levels of serum TG, phospholipids, enhanced systemic oxidative stress and inflammatory response in few healthy subjects as well as in subjects with established CHD. These findings suggest that the functional loss in HDL might arise as an adaptive response to acute phase state, but when persistently expressed this dysfunctional HDL can induce chronic metabolic changes that drive further atherogenesis, eventually negatively impacting clinical outcomes. Further research involving more healthy subjects are needed to confirm these findings and to explore the factors causing dysfunctionality in HDL.

There is now a consensus that atherosclerosis represents a state of chronic inflammation of the vascular wall[17]. Since oxidative stress and ROS formation have been implicated in various pathological conditions involving cardiovascular disease, cancer, neurological disorders, diabetes, ischemia,other diseases and ageing [49], we determined whether dysfunctional HDL influence the production of 
ROS in monocytes/macrophages, the predominant cells involved in atherogenesis. This study demonstrated that dysfunHDL induced intracellular ROS formation in these cells in a concentration dependent manner, indicating its pro-oxidant nature. This was in contrast to the effect of funHDL, where HDL in culture inhibited excess ROS production and confirming its antioxidative functionality. This finding confirm our previous data that oxidized- HDL induces pro-inflammatory response in these cells[13]. Hence, the prevalence of functionally deficient HDL might induce cellular pro-oxidative response as one mechanism of atherogenesis. Since the proinflammatory potential of HDL appears to be reversible[2],strategies that improve the antioxdative/anti-inflammatory properties of HDL,including dietary, lifestyle factors, and/ or pharmacologic approaches, may be the most effective additional treatment for reducing cardiovascular risk. The complexity of HDL structure and function demands more investigation to better understand HDL metabolism, function and its regulation in humans. Our observations suggests that alterations in HDL composition might enhance the formation of inflammatory particles, and hence quantifying the HDL function could help identify novel anti-inflammatory and cardioprotective actions of HDL and provide insights into lipid therapy.

\section{Conclusion}

In conclusion, this study demonstrates the prevalence of dysfunHDL phenotype even among healthy subjects, despite normal HDL-C level, as well as in most of subjects with known CHD. This dysfunctional HDL is not cardioprotective, but is pro-oxidant as it induces cellular oxidative stress that can enhance vascular inflammation and atherogenesis. Functional assay of HDL could lead to improved predictive accuracy for cardiovascular disease risk associated with circulating HDL. Raising HDL's atheroprotective function may represent an additional therapeutic target for reducing high cardiovascular risk.

\section{Acknowledgments}

The authors wish to thank the Director of Sree Chitra Tirunal Institute for Medical Sciences and Technology, Thiruvananthapuram, Kerala, India, for providing necessary facilities to carryout the work. Junior research fellowship from INSPIRE, DST, New Delhi, granted to Sini.S. is gratefully acknowledged. Authors thank Dr.Harikrishnan for kindly providing few samples from patients with established CHD for comparitive studies.

Conflict of interest: The authors declare no conflict of interest.

\section{References}

[1] N. Ahmad, R. Bhopal, Is coronary heart disease rising in India? A systematic review based on ECG defined coronary heart disease, Heart, 91 (2005) 719-725.
[2] G.K. Hansson, Inflammation, atherosclerosis, and coronary artery disease, N Engl J Med, 352 (2005) 1685-1695.

[3] P.J. Barter, S. Nicholls, K.A. Rye, G.M. Anantharamaiah, M. Navab, A.M. Fogelman, Antiinflammatory properties of HDL, Circ Res, 95 (2004) 764-772.

[4] P.P. Toth, High-density lipoprotein and cardiovascular risk, Circulation, 109 (2004) 1809-1812.

[5] A.M. Gotto, Jr., E.A. Brinton, Assessing low levels of highdensity lipoprotein cholesterol as a risk factor in coronary heart disease: a working group report and update, J Am Coll Cardiol, 43 (2004) 717-724.

[6] H.B. G, V.S. Rao, V.V. Kakkar, Friend Turns Foe: Transformation of Anti-Inflammatory HDL to Proinflammatory HDL during Acute-Phase Response, Cholesterol, 2011 (2011) 274629.

[7] A.A. Baron, S.B. Baron, High levels of HDL cholesterol do not predict protection from cardiovascular disease in women, Prev Cardiol, 10 (2007) 125-127.

[8] E. Eren, N. Yilmaz, O. Aydin, High Density Lipoprotein and it's Dysfunction, Open Biochem J, 6 (2012) 78-93.

[9] F. Paneni, F. Cosentino, F. Marrara, F. Palano, G. Capretti, M. Gregori, G. Tocci, M. Testa, M. Volpe, The clinical relevance of dysfunctional HDL in patients with coronary artery disease: a 3-year follow-up study, Int J Cardiol, 158 (2012) 158-160.

[10] S. Dodani, Excess coronary artery disease risk in South Asian immigrants: can dysfunctional high-density lipoprotein explain increased risk?, Vasc Health Risk Manag, 4 (2008) 953-961.

[11] M. McMahon, J. Grossman, B. Skaggs, J. Fitzgerald, L. Sahakian, N. Ragavendra, C. Charles-Schoeman, K. Watson, W.K. Wong, E. Volkmann, W. Chen, A. Gorn, G. Karpouzas, M. Weisman, D.J. Wallace, B.H. Hahn, Dysfunctional proinflammatory high-density lipoproteins confer increased risk of atherosclerosis in women with systemic lupus erythematosus, Arthritis Rheum, 60 (2009) 2428-2437.

[12] R. Asleh, S. Blum, S. Kalet-Litman, J. Alshiek, R. MillerLotan, R. Asaf, W. Rock, M. Aviram, U. Milman, C. Shapira, Z. Abassi, A.P. Levy, Correction of HDL dysfunction in individuals with diabetes and the haptoglobin 2-2 genotype, Diabetes, 57 (2008) 2794-2800.

[13] V.S. Soumyarani, N. Jayakumari, Oxidatively modified high density lipoprotein promotes inflammatory response in human monocytes-macrophages by enhanced production of ROS, TNF-alpha, MMP-9, and MMP-2, Mol Cell Biochem, 366 (2012) 277-285

[14] N. Jayakumari, G. Thejaseebai, High prevalence of low serum paraoxonase- 1 in subjects with coronary artery disease, J Clin Biochem Nutr, 45 (2009) 278-284.

[15] H. Mirjafari, A. Al-Husain, I.N. Bruce, Cardiovascular risk factors in inflammatory arthritis, Curr Opin Lipidol, 22 (2011) 296-301.

[16] E. Regidor, J. Franch, M. Segui, R. Serrano, F. RodriguezArtalejo, S. Artola, Traditional Risk Factors Alone Could Not Explain the Excess Mortality in Patients With Diabetes: A national cohort study of Spanish older adults, Diabetes Care, (2012). 
[17] R. Ross, Atherosclerosis--an inflammatory disease, N Engl J Med, 340 (1999) 115-126.

[18] P. Libby, Inflammation in atherosclerosis, Nature, 420 (2002) 868-874.

[19] E. Zouridakis, P. Avanzas, R. Arroyo-Espliguero, S. Fredericks, J.C. Kaski, Markers of inflammation and rapid coronary artery disease progression in patients with stable angina pectoris, Circulation, 110 (2004) 1747-1753.

[20] P.M. Ridker, C.H. Hennekens, B. Roitman-Johnson, M.J. Stampfer, J. Allen, Plasma concentration of soluble intercellular adhesion molecule 1 and risks of future myocardial infarction in apparently healthy men, Lancet, 351 (1998) 8892.

[21] A.T. Remaley, M. Amar, D. Sviridov, HDL-replacement therapy: mechanism of action, types of agents and potential clinical indications, Expert Rev Cardiovasc Ther, 6 (2008) 1203-1215.

[22] C. Izzo, F. Grillo, E. Murador, Improved method for determination of high-density-lipoprotein cholesterol I. Isolation of high-density lipoproteins by use of polyethylene glycol 6000, Clin Chem, 27 (1981) 371-374.

[23] G. Gallou, A. Ruelland, B. Legras, D. Maugendre, H. Allannic, L. Cloarec, Plasma malondialdehyde in type 1 and type 2 diabetic patients, Clin Chim Acta, 214 (1993) 227234.

[24] N. Jayakumari, V. Ambikakumari, K.G. Balakrishnan, K.S. Iyer, Antioxidant status in relation to free radical production during stable and unstable anginal syndromes, Atherosclerosis, 94 (1992) 183-190.

[25] S.T. Omaye, J.D. Turnbull, H.E. Sauberlich, Selected methods for the determination of ascorbic acid in animal cells, tissues, and fluids, Methods Enzymol, 62 (1979) 3-11.

[26] M. Toth, A. Sohail, R. Fridman, Assessment of gelatinases (MMP-2 and MMP-9) by gelatin zymography, Methods Mol Biol, 878 (2012) 121-135.

[27] M.J. Chapman, S. Goldstein, D. Lagrange, P.M. Laplaud, A density gradient ultracentrifugal procedure for the isolation of the major lipoprotein classes from human serum, J Lipid Res, 22 (1981) 339-358.

[28] J.R. Dulley, P.A. Grieve, A simple technique for eliminating interference by detergents in the Lowry method of protein determination, Anal Biochem, 64 (1975) 136-141.

[29] K. Lorentz, B. Flatter, E. Augustin, Arylesterase in serum: elaboration and clinical application of a fixed-incubation method, Clin Chem, 25 (1979) 1714-1720.

[30] M. Navab, S.Y. Hama, G.P. Hough, G. Subbanagounder, S.T Reddy, A.M. Fogelman, A cell-free assay for detecting HDL that is dysfunctional in preventing the formation of or inactivating oxidized phospholipids, J Lipid Res, 42 (2001) $1308-1317$

[31] S. Jovinge, M.P. Ares, B. Kallin, J. Nilsson, Human monocytes/macrophages release TNF-alpha in response to OxLDL, Arterioscler Thromb Vasc Biol, 16 (1996) 1573-1579.

[32] C. Charles-Schoeman, J. Watanabe, Y.Y. Lee, D.E. Furst, S. Amjadi, D. Elashoff, G. Park, M. McMahon, H.E. Paulus, A.M. Fogelman, S.T. Reddy, Abnormal function of high- density lipoprotein is associated with poor disease control and an altered protein cargo in rheumatoid arthritis, Arthritis Rheum, 60 (2009) 2870-2879.

[33] J.M. Lee, R.P. Choudhury, Atherosclerosis regression and high-density lipoproteins, Expert Rev Cardiovasc Ther, 8 (2010) 1325-1334.

[34] M. Navab, S.T. Reddy, B.J. Van Lenten, A.M. Fogelman, HDL and cardiovascular disease: atherogenic and atheroprotective mechanisms, Nat Rev Cardiol, 8 (2011) 222-232.

[35] B.J. Ansell, M. Navab, S. Hama, N. Kamranpour, G. Fonarow, G. Hough, S. Rahmani, R. Mottahedeh, R. Dave, S.T. Reddy, A.M. Fogelman, Inflammatory/antiinflammatory properties of high-density lipoprotein distinguish patients from control subjects better than high-density lipoprotein cholesterol levels and are favorably affected by simvastatin treatment, Circulation, 108 (2003) 2751-2756.

[36] S. Manzi, E.N. Meilahn, J.E. Rairie, C.G. Conte, T.A. Medsger, Jr., L. Jansen-McWilliams, R.B. D'Agostino, L.H. Kuller, Age-specific incidence rates of myocardial infarction and angina in women with systemic lupus erythematosus: comparison with the Framingham Study, Am J Epidemiol, 145 (1997) 408-415.

[37] M. Navab, G.M. Ananthramaiah, S.T. Reddy, B.J. Van Lenten, B.J. Ansell, S. Hama, G. Hough, E. Bachini, V.R. Grijalva, A.C. Wagner, Z. Shaposhnik, A.M. Fogelman, The double jeopardy of HDL, Ann Med, 37 (2005) 173-178.

[38] T. Itoh, H. Matsuda, M. Tanioka, K. Kuwabara, S. Itohara, R. Suzuki, The role of matrix metalloproteinase-2 and matrix metalloproteinase- 9 in antibody-induced arthritis, J Immunol, 169 (2002) 2643-2647.

[39] Z.S. Galis, J.J. Khatri, Matrix metalloproteinases in vascular remodeling and atherogenesis: the good, the bad, and the ugly, Circ Res, 90 (2002) 251-262.

[40] E. Rodrigo, M. Lopez-Hoyos, R. Escallada, G. FernandezFresnedo, J.C. Ruiz, C. Pinera, J.G. Cotorruelo, J.A. Zubimendi, A.L. de Francisco, M. Arias, Circulating levels of matrix metalloproteinases MMP-3 and MMP-2 in renal transplant recipients with chronic transplant nephropathy, Nephrol Dial Transplant, 15 (2000) 2041-2045.

[41] S. Takeshita, T. Tokutomi, H. Kawase, K. Nakatani, H. Tsujimoto, Y. Kawamura, I. Sekine, Elevated serum levels of matrix metalloproteinase-9 (MMP-9) in Kawasaki disease, Clin Exp Immunol, 125 (2001) 340-344.

[42] P. Kampus, P. Muda, J. Kals, T. Ristimae, K. Fischer, R. Teesalu, M. Zilmer, The relationship between inflammation and arterial stiffness in patients with essential hypertension, Int J Cardiol, 112 (2006) 46-51.

[43] J.D. Smith, Dysfunctional HDL as a diagnostic and therapeutic target, Arterioscler Thromb Vasc Biol, 30 (2010) 151155.

[44] N. Yilmaz, Relationship between paraoxonase and homocysteine: crossroads of oxidative diseases, Arch Med Sci, 8 (2012) 138-153.

[45] M. Navab, S.Y. Hama, C.J. Cooke, G.M. Anantharamaiah, M. Chaddha, L. Jin, G. Subbanagounder, K.F. Faull, S.T. Reddy, N.E. Miller, A.M. Fogelman, Normal high density lipoprotein inhibits three steps in the formation of mildly oxidized low density lipoprotein: step 1, J Lipid Res, 41 
(2000) 1481-1494.

[46] M. Navab, S.Y. Hama, G.M. Anantharamaiah, K. Hassan, G.P. Hough, A.D. Watson, S.T. Reddy, A. Sevanian, G.C. Fonarow, A.M. Fogelman, Normal high density lipoprotein inhibits three steps in the formation of mildly oxidized low density lipoprotein: steps 2 and 3, J Lipid Res, 41 (2000) 1495-1508.

[47] D.L. Sparks, W.S. Davidson, S. Lund-Katz, M.C. Phillips, Effects of the neutral lipid content of high density lipoprotein on apolipoprotein A-I structure and particle stability, J Biol Chem, 270 (1995) 26910-26917.
[48] V. Zago, S. Sanguinetti, F. Brites, G. Berg, J. Verona, F. Basilio, R. Wikinski, L. Schreier, Impaired high density lipoprotein antioxidant activity in healthy postmenopausal women, Atherosclerosis, 177 (2004) 203-210.

[49] D. Deepa, N. Jayakumari, S.V. Thomas, Oxidative stress is increased in women with epilepsy: Is it a potential mechanism of anti-epileptic drug-induced teratogenesis?, Ann Indian Acad Neurol, 15 (2012) 281-286. 